'Departamento de Medicina, Campus Centro, Facultad de Medicina, Universidad de Chile, Hospital Clínico San Borja Arriarán, Academia Chilena de Medicina. Santiago, Chile. Laboratorio Clínico, Hospital Clínico de la Universidad de Chile, Academia Chilena de Medicina. Santiago, Chile. ${ }^{3}$ Departamento de Pediatría, Campus Oriente, Facultad de Medicina, Universidad de Chile. Hospital Luis Calvo Mackenna, Academia Chilena de Medicina. Santiago, Chile.

${ }^{4}$ Centro de Epidemiología y

Políticas de Salud, Facultad de Medicina Clínica Alemana Universidad del Desarrollo. Santiago, Chile.

${ }^{5}$ Consultora independiente.

Todos los autores tuvieron el mismo grado de participación y responsabilidad en el pronunciamiento

Convocante: Prof. Dr. Rodolfo Armas M., Presidente, Academia Chilena de Medicina.

Trabajo no recibió financiamiento. Los autores declaran no tener conflictos de interés.

Recibido el 11 de marzo de 2020 aceptado el 8 de junio de 2020

Correspondencia: Marcelo Wolff Almirante Montt 453. Santiago, Chile. marcewolff@yahoo.com

\section{Evaluación de la infección por VIH en Chile: pronunciamiento del Comité VIH de la Academia Chilena de Medicina}

\author{
MARCELO WOLFF R. ${ }^{1}$, MARÍA EUGENIA PINTO C. ${ }^{2}$, \\ MARÍA ELENA SANTOLAYA D. ${ }^{3}$, XIMENA AGUILERA S. ${ }^{4}$, \\ RAQUEL CHILD G. ${ }^{5}$
}

\section{Evaluation of HIV epidemic in Chile. A statement of the Chilean Academy of Medicine}

The Chilean Academy of Medicine convened a commission to evaluate the status of HIV epidemic and the national response to it, regarding its achievements, gaps and challenges, aiming to recommend actions to optimize assessment quality and national response. This publication summarizes the agreed upon opinion of its members. The epidemic is overwhelmingly sexually transmitted, predominant in homo/bisexual men. Vertical transmission is very low. An increasing number of new diagnoses is occurring, with relative over representation of foreign people lately. There is a legal guarantee of confidentiality, nondiscrimination and treatment for those affected, both in the private and public sector. All public health services have active HIV care units. Modern antiviral drugs and monitoring tests are also available. Despite these clear achievements, insufficient, occasionally inadequate public policies and certain rigid regulations thwart optimal effectivity and efficiency of the programs, contributing to the slow and incomplete compliance with international commitments. Shortcomings worth highlighting are: suboptimal educational and preventive programs directed to youngsters, vulnerable and general population; persistent underdiagnosis of infected population; cumbersome requirements to request and inform diagnostic tests, thus discouraging testing; excessive centralization and long latency of diagnosis confirmation and monitoring tests; incomplete epidemiologic analysis and public reporting of findings; non flexibility and slow updating of therapeutic guidelines; insufficient adaptation of care and drug delivery modalities to patients' needs; excessive administrative requirements at care centers and restrictive legislation for outcome and interventional clinical research. Recommendations to deal with these issues were proposed.

(Rev Med Chile 2020; 148: 818-821)

Key words: Chile; Epidemics; HIV Infections.

\section{Consideraciones generales y objetivos}

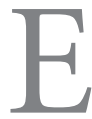
Comité Consultivo de VIH de la Academia Chilena de Medicina ha tenido por objeto elaborar un documento consensuado entre sus miembros en donde, basado en aspectos epidemiológicos, fisiopatológicos, clínicos y terapéuti- cos generales y globales, analice la situación de esta epidemia en el país desde su desarrollo reciente a la situación actual, así como expectativas futuras respecto a fortalezas, logros, brechas, desafíos y recomendaciones en su enfrentamiento, con el fin de contribuir a optimizar las áreas de educación, prevención, diagnóstico individual y poblacional, 
terapia, inclusión y no discriminación que ayuden a disminuir, mitigar y controlar la epidemia en el país, con beneficio no solo para los afectados, sino para toda la sociedad.

Los planteamientos se elaboraron durante sesiones de trabajo periódicas durante 2019, tomando en cuenta la información nacional y global vigente hasta fines de ese período. Esta publicación representa resumidamente las ideas centrales de su análisis y recomendaciones. Al documento en su integridad se puede acceder en la página electrónica oficial de la Academia Chilena de Medicina (www.academiachilenademedicina.cl) y en la edición 2020 de su boletín oficial, junto con las referencias que lo sustentan.

Este documento representa la opinión consensuada de sus autores y, si bien fue convocada en el seno de la Academia Chile de Medicina del Instituto de Chile, no es un planteamiento oficial de la institución

\section{Resumen ejecutivo}

La infección por el virus de la inmunodeficiencia humana (VIH) se mantiene como uno de los problemas de salud más importante del mundo, a pesar de la existencia de una terapia que, sin ser curativa, controla la enfermedad, mejora la calidad de vida y reduce la infectividad de las personas tratadas. Esta terapia, actualmente recomendada universalmente a todas las personas infectadas apenas sean diagnosticadas, está en expansiva aplicación a nivel global, pero aún hay millones de personas que no están diagnosticadas y otras que se siguen infectando. El número de personas que viven con VIH sigue aumentando globalmente por la persistente aparición de nuevos casos y la marcada reducción en letalidad de los previamente infectados que reciben tratamiento.

Chile no está ajeno al problema, el que localmente tiene características propias: afecta principalmente a adultos jóvenes en etapas laboralmente productivas, es de transmisión sexual en más de $95 \%$ de los casos, no más de $15 \%$ es en mujeres y la población masculina homo/bisexual representa la mayoría de los casos; la transmisión por drogadicción endovenosa es excepcional y la transmisión vertical es muy baja, gracias a la obligatoriedad legal de ofrecer el examen diagnóstico a toda embarazada en el país y a la efectiva prevención de transmisión perinatal en base a oportuno tratamiento antirretroviral en aquellas infectadas, que ha llevado a una mínima y lentamente decreciente población infantil que vive con VIH. La incidencia de la infección ha experimentado incremento significativo en el último quinquenio en base a nuevos diagnósticos reportados por el único centro confirmatorio nacional, destacando en el período el aumento de la proporción de población extranjera en el total de estos diagnósticos, que en los primeros 11 meses de 2019 llegaba a 40\%. Actualmente, se considera que la prevalencia es de $0,5 \times 10^{5}$ en la población de 15 a 49 años. En el país, el tratamiento de la patología está cubierta por las garantías de la ley AUGE, que cubre medicación y monitoreo tanto en el sector público -que abarca el $85 \%$ de los casos- como en el privado, con plazos de cumplimiento y garantía financiera. El país ha tenido una respuesta legislativa, administrativa y sanitaria que resguarda la confidencialidad, la no discriminación, la pesquisa (en embarazadas) y, actualmente, el tratamiento antirretroviral universal inmediato. Se han implementado centros de atención especializada en todos los servicios de salud del país. Se estimaba oficialmente que a fines de 2017, 72\% de la población infectada había sido diagnosticada, $83 \%$ de ella recibía terapia y $90 \%$ de esta estaba en tratamiento exitoso con supresión de la replicación viral (54\% del total de infectados y sin transmisibilidad a terceros). Los 2 primeros resultados estaban por debajo de la meta global recomendada para 2020 por la división de la Organización de las Naciones Unidas directamente encargada del enfrentamiento de la pandemia (ONUSIDA) de 90\%, 90\%, 90\%, respectivamente. La autoridad sanitaria ha reportado extraoficialmente que para mediados de 2019 estos porcentajes habían mejorado. La mortalidad de la enfermedad se mantiene por sobre la meta propuesta de la Organización Mundial de la Salud (OMS) $\left(1,2 \times 10^{5}\right)$, a pesar de que la letalidad ha disminuido marcadamente con el tratamiento. El gasto país en el manejo de la epidemia es considerable (alrededor de US\$ 160 millones en sistema público de salud en 2018, según la autoridad ministerial, con $1 / 3$ adicional en el sistema privado) y en constante aumento, mayoritariamente en medicamentos. La investigación nacional en patología VIH se ha desarrollado lentamente, y abarca las áreas de epidemiología, 
aspectos psicosociales, clínica y virología básica, algunas de estas de cooperación internacional.

En opinión de este comité, el enfoque nacional sobre la epidemia de VIH en el país ha implicado un importante compromiso político de diversos gobiernos, llevando a logros trascendentes en el manejo de la patología. Sin embargo, también hay que constatar insuficiencias, imperfecciones y carencias en ciertas políticas públicas que han dificultado optimizar los reconocibles logros generales, especialmente en las áreas de educación, prevención, expansión y simplificación diagnóstica e investigación clínico epidemiológica. El país está teniendo dificultades para cumplir en los plazos adecuados con los compromisos adquiridos a nivel internacional sobre el manejo de la epidemia tanto respecto a la meta 90-90-90, como en la reducción de mortalidad por VIH que actualmente es más del doble de lo comprometido para el año 2020.

Entre los logros a destacar en el enfrentamiento nacional a la epidemia se distinguen:

- Incorporación de la patología a las garantías de la ley AUGE, tanto en terapia como monitoreo.

- Adecuada tecnología diagnóstica y de monitoreo del tratamiento con homogenización del proceso.

- Legislación y reglamentación que garantiza, desde el proceso diagnóstico al terapéutico, la confidencialidad de la información y la no discriminación.

- Disponibilidad de centros de atención especializada en todos los servicios de salud públicos del país.

- Disponibilidad de medicación moderna efectiva y de buena calidad.

- Normativa obligatoria nacional de pesquisa de infección en embarazadas y garantía de medidas preventivas de transmisión vertical en infectadas.

- Disminución de la trasmisión vertical a menos de $2 \%$.

Sin embargo, se aprecian brechas importantes que pueden mejorar:

- Insuficiente y subóptima elaboración e implementación de programas de educación sexual general y de prevención de infecciones de transmisión sexual (ITS), que sean de carácter estable e incorporados a los programas y obligatorios en establecimientos educacionales de nivel primario a superior.
- Insuficientes y subóptimas campañas de prevención de infección por VIH e ITS para la población general y grupos de riesgo aumentado.

- Débil política proactiva de expansión diagnóstica poblacional que mantiene brecha importante entre población infectada y la diagnosticada.

- Legislación y reglamentación profusa y compleja, e incluso punitiva, que desincentiva una adecuada y mayor solicitud diagnóstica por parte de los profesionales de salud.

- Exceso de centralización de los procesos de confirmación diagnóstica y monitoreo terapéutico.

- Falta de mayor evaluación epidemiológica de la epidemia, su evolución y las causas de estos cambios, así como socialización de esta información.

- Excesiva recarga administrativa, muchas veces redundantes, en los centros de atención.

- Falta de ajuste oportuno en infraestructura y recurso humano de acuerdo a evolución de la carga asistencial en los centros públicos de atención.

- Falta de apoyo del Estado para el fomento y soporte económico para la investigación multiprofesional y traslacional en patología VIH.

- Legislación reciente restrictiva y limitante para la investigación clínica intervencional.

- Discordancia frecuente entre los datos nacionales reportados localmente y los de organismos sanitarios internacionales sobre la epidemia, sus logros y carencias, dificultando una adecuada interpretación de la situación nacional.

- Poca flexibilidad del sistema público para los horarios de atención, los períodos de entrega de medicamentos y la realización de exámenes para una población mayormente activa en lo laboral.

- Poca flexibilidad de las normativas ministeriales para ajustar oportunamente las guías terapéuticas, incluidos los frecuentes ajustes conceptuales de la terapia y los nuevos medicamentos, ya que están sujetos a modificaciones de leyes y no de reglamentos.

Los miembros del comité, convocados por la Academia Chilena de Medicina, consideran que el país ha realizado un gran esfuerzo para combatir la epidemia. Este esfuerzo puede optimizar 
sus frutos con ajustes factibles, especialmente dentro del sector público, por lo que se permite proponer las siguientes recomendaciones generales:

- Fortalecer el compromiso político y adecuar el marco legal.

- Promover estrategias de cambio de conductas basadas en la evidencia científica, la educación y las campañas preventivas.

- Reducir las barreras de acceso al diagnóstico y mejorar sus oportunidades, fomentar su realización, tanto por iniciativas públicas generales, como en la atención sanitaria individual cotidiana.

- Aumentar por ley la oferta de examen diagnóstico a más de un test en embarazadas, con uno de ellos realizado en el tercer trimestre del embarazo.

- Descentralizar procesos diagnósticos y de monitoreo de tratamiento a lo largo del país, manteniendo una coordinación central.

- Mejorar el análisis estadístico y epidemiológico para desarrollar estrategias adecuadas, incluyendo el impacto de la población extranjera migrante en la epidemia nacional.

- Optimizar la calidad de los datos entregados a los organismos internacionales.

- Adecuar oportunamente infraestructura y recurso humano de acuerdo a la evolución de la epidemia.

- Simplificar el monitoreo y minimizar la redundancia de reportes administrativos.

- Flexibilizar las políticas de compra, incorporación de nuevos medicamentos y la oportunidad de actualización de las guías terapéuticas.

- Flexibilizar los períodos de entrega de medicamentos a los pacientes de acuerdo a sus necesidades y obligaciones laborales o educacionales.

- Fomentar como Estado la investigación clínico epidemiológica y de ciencias básicas en VIH y sus consecuencias.

- Incrementar la participación social en la toma de decisiones. 